\title{
Greater ultrasonographic changes in pediatric moyamoya patients compared with adults after indirect revascularization surgeries
}

\author{
Shin-Joe Yeh, MD, MS, ${ }^{1}$ Sung-Chun Tang, MD, PhD, ${ }^{1}$ Li-Kai Tsai, MD, PhD, ${ }^{1}$ \\ Chung-Wei Lee, MD, PhD, ${ }^{2}$ Ya-Fang Chen, MD, ${ }^{2}$ Hon-Man Liu, MD, PhD, ${ }^{3,4}$ \\ Shih-Hung Yang, MD, PhD, ${ }^{5}$ Yu-Lin Hsieh, BS, ${ }^{1}$ Meng-Fai Kuo, MD, PhD, ${ }^{5}$ and \\ Jiann-Shing Jeng, MD, PhD'
}

\begin{abstract}
${ }^{1}$ Stroke Center and Department of Neurology, and Departments of ${ }^{2}$ Medical Imaging and ${ }^{5}$ Neurosurgery, National Taiwan University Hospital, Taipei; ${ }^{3}$ Department of Radiology, Fu Jen Catholic University Hospital; and ${ }^{4}$ School of Medicine, Fu Jen University, New Taipei City, Taiwan
\end{abstract}

\begin{abstract}
OBJECTIVE Pediatric and adult patients with moyamoya disease experience similar clinical benefits from indirect revascularization surgeries, but there are still debates about age-related angiographic differences of the collaterals established after surgery. The goal of this study was to assess age-related differences on ultrasonography before and after indirect revascularization surgeries in moyamoya patients, focusing on some ultrasonographic parameters known to be correlated with the collaterals supplied by the external carotid artery (ECA).
\end{abstract}

METHODS The authors prospectively included moyamoya patients (50 and 26 hemispheres in pediatric and adult patients, respectively) who would undergo indirect revascularization surgery. Before surgery and at 1, 3, and 6 months after surgery, the patients underwent ultrasonographic examinations. The ultrasonographic parameters included peak-systolic velocity (PSV), end-diastolic velocity (EDV), resistance index (RI), and flow volume (FV) measured in the ECA, superficial temporal artery (STA), and internal carotid artery on the operated side. The mean values, absolute changes, and percentage changes of these parameters were compared between the pediatric and adult patients. Logistic regression analysis was used to clarify the determinants affecting postoperative EDV changes in the STA.

RESULTS Before surgery, the adult patients had mean higher EDV and lower RI in the STA and ECA than the pediatric group (all $p<0.05$ ). After surgery, the pediatric patients had greater changes (absolute and percentage changes) in the PSV, EDV, RI, and FV in the STA and ECA (all $p<0.05$ ). The factors affecting postoperative EDV changes in the STA at 6 months were age $(p=0.006)$ and size of the revascularization area (i.e., revascularization in more than the temporal region vs within the temporal region; $p=0.009$ ). Pediatric patients who received revascularization procedures in more than the temporal region had higher velocities (PSV and EDV) in the STA than those who received revascularization within the temporal region ( $p<0.05$ at $1-6$ months), but such differences were not observed in the adult group.

CONCLUSIONS The greater changes of these parameters in the STA and ECA in pediatric patients than in adults after indirect revascularization surgeries indicated that pediatric patients might have a greater increase of collaterals postoperatively than adults. Pediatric patients who undergo revascularization in more than the temporal region might have more collaterals than those who undergo revascularization within the temporal region.

https://thejns.org/doi/abs/10.3171/2018.6.PEDS18151

KEYWORDS moyamoya disease; age; indirect revascularization; ultrasonography; collaterals; vascular disorders

$\mathrm{M}$ OYAMOYA disease (MMD) is a special cause of stroke that is characterized by chronic progressive stenosis of major intracranial arteries with gradual development of multiple tiny collaterals for compensation. ${ }^{9}$ Its importance lies not only in its higher preva- lence in pediatric patients but also its progressive course and treatability. ${ }^{11}$ Epidemiological studies have shown that age affects the presenting stroke subtypes of MMD. Although the major stroke subtype is ischemic stroke in both age groups with MMD, hemorrhagic stroke is more

ABBREVIATIONS ECA = external carotid artery; EDAS = encephaloduroarteriosynangiosis; EDS = encephalodurosynangiosis; EDV = end-diastolic velocity; EMS = encephalomyosynangiosis; EPS = encephalopericraniosynangiosis; FV = flow volume; ICA = internal carotid artery; MMD = moyamoya disease; PSV = peak-systolic velocity; RI = resistance index; STA = superficial temporal artery.

SUBMITTED March 10, 2018. ACCEPTED June 5, 2018

INCLUDE WHEN CITING Published online August 31, 2018; DOI: 10.3171/2018.6.PEDS18151. 
common in adult moyamoya patients than in the pediatric population. ${ }^{2,9}$ Hemorrhagic stroke in adult moyamoya patients is related to more advanced MMD stages and more collaterals?

Indirect revascularization surgeries can be performed to treat hypoperfusion in both pediatric and adult moyamoya patients by inducing the development of transpial and transdural collaterals. ${ }^{5,13}$ Clinically, both age groups experience benefits from indirect revascularization surgeries without a definite age-related difference in the rate of absence of new ischemic symptoms or in modified Rankin Scale score. ${ }^{4,6}$ However, quantitative evidence for any age-related difference in the surgery-induced collaterals is limited. Some studies have shown that the neovascularization after indirect revascularization surgery was more extensive in pediatric patients than in adults, while other reports did not show a significant age-related difference. ${ }^{3,4,17,19}$ Of note, these previous studies were all assessed using cerebral angiography. Although angiography is the gold-standard examination tool for MMD, there is some difficulty in quantifying the hemodynamics of vessels by angiography.

Ultrasound is an alternative tool for the quantitative assessment of the cerebral hemodynamics after indirect revascularization surgeries. ${ }^{14,15,18}$ Given that these postoperative collaterals are supplied by branches of the external carotid artery (ECA), especially the superficial temporal artery (STA), our previous study showed that better collateral establishment on angiography was significantly correlated with a higher end-diastolic velocity (EDV), lower resistance index (RI), and larger flow volume (FV) in the STA and ECA on color Doppler ultrasonography. ${ }^{18}$ However, ultrasonography has never been used to compare the hemodynamics between pediatric and adult moyamoya patients after indirect revascularization surgery. Our hypothesis was that pediatric moyamoya patients might have more obvious hemodynamic changes in the STA and ECA after indirect revascularization surgery than adult patients. Therefore, this study compared the ultrasonographic parameters between pediatric and adult moyamoya patients before and after indirect revascularization surgeries and analyzed the factors affecting the postoperative changes of EDV in the STA, which might be a potential indicator for the extent of collaterals. ${ }^{18}$

\section{Methods}

\section{Patient Populations}

Patients with suspected MMD underwent cerebral angiography to confirm MMD and brain MR perfusion imaging to examine areas of hypoperfusion. Pediatric patients underwent indirect revascularization surgeries, while adult patients were able to choose direct, indirect, or combined direct and indirect revascularization surgery after receiving detailed information about the benefits and risks of the different surgeries. Consecutive moyamoya patients who were admitted to the National Taiwan University Children's Hospital between August 2012 and April 2017, diagnosed by cerebral angiography, and were planned for indirect revascularization surgeries were prospectively enrolled into this follow-up study. All indirect revascularization procedures were performed by a single surgeon (M.F.K.), and there was no difference in the surgical technique of the same procedure between the adult and pediatric groups. Only the brain areas with evidence of hypoperfusion on MR perfusion imaging received revascularization procedures. Encephaloduroarteriosynangiosis (EDAS) and encephalomyosynangiosis (EMS) were performed in the temporal region. Encephalopericraniosynangiosis (EPS) was performed in other hypoperfusion regions, mostly the frontal area. Encephalodurosynangiosis (EDS) was performed in every indirect revascularization procedure to facilitate additional synangiosis in each craniotomy. The protocol of this follow-up study included a series of examinations, including ultrasonography, MRI, and angiography (Fig. 1). The patients who completed preoperative and postoperative serial ultrasonographic examinations were included. Patients were divided into pediatric ( $<20$ years old) and adult ( $\geq 20$ years old) groups. This study was approved by the research ethics committee of the National Taiwan University Hospital, and all of the included patients and the children's parents gave their informed consent.

\section{Ultrasonographic Examination}

Carotid Doppler ultrasonographic examination was performed using a color-coded ultrasound system (iE33, Philips Medical Systems) with an 11- to 3-MHz lineararray transducer. The examination was performed before surgery and at 1,3 , and 6 months after surgery. This examination followed the protocol of that described in a previous report and was performed by a single technician who specialized in carotid Doppler ultrasonography. ${ }^{18}$ The sonographic parameters included peak-systolic velocity (PSV), EDV, RI, and FV measured in the STA, ECA, and internal carotid artery (ICA). The STA was analyzed at the common STA segment at ear level. The FV of each artery was calculated using the ultrasound machine from the time-averaged mean flow velocity multiplied by the cross-sectional area, which was calculated from the diameter. The diameter of each vessel was measured between the inner luminal walls at the end-diastolic state. The measurement was performed once for each vessel because pediatric patients could not tolerate prolonged examination.

\section{Statistical Analysis}

Categorical variables are shown as percentages, and continuous variables are presented as the mean \pm standard deviation. The Fisher's exact test and Mann-Whitney Utest were used to compare categorical variables and continuous variables between the 2 age groups, respectively. First, we compared the hemodynamic parameters between the 2 age groups at different time points using the MannWhitney U-test. Second, as the postoperative absolute changes (postoperative value - preoperative value) of each parameter may be closely related to the hemodynamic changes, the absolute changes were compared between the 2 age groups at consecutive time points using the MannWhitney U-test. Third, similar absolute changes based on different preoperative values may have a different impact. Thus, percentage changes (absolute change/preoperative 
value $\times 100 \%$ ) between the 2 age groups were compared using the Mann-Whitney U-test. Fourth, the diameters of the 3 vessels were also compared between the 2 age groups at consecutive time points in order to clarify the contribution of diameter to the difference in flow volume between the 2 age groups using the Mann-Whitney U-test. To determine the factors affecting the absolute changes of EDV in the STA, logistic regression analysis was performed to evaluate the influence of age, sex, Suzuki stage, preoperative EDV, and surgical procedures. The surgical procedures were divided into 2 sets according to the revascularization area within the temporal region (EDAS only, EDAS + EMS) and more than the temporal region (EDAS + EPS). Mutual adjustment was carried out in the multivariate analysis of the logistic regression model. We also separately compared the influences of procedures on the hemodynamic parameters in children and adults using the Mann-Whitney U-test.

\section{Results}

\section{Two Representative Cases in the Different Age Groups}

The preoperative and postoperative ultrasonographic results for the STA on the operative side in 2 patients of different ages are shown in Fig. 2. In the first case, an 8-year-old girl with bilateral MMD received EDAS surgery on the left side (Fig. 2 upper). The preoperative ultrasonographic examinations of the left STA showed a highresistance pattern. At the 1-month postoperative visit, there was a larger increase in the EDV (380\% increase) than in the PSV (114\% increase) and a decrease in the RI (24\% decrease), turning the high-resistance flow pattern into a low-resistance pattern.

In the second case, a 30-year-old woman with bilateral MMD received EDAS + EPS on the right side (Fig. 2 lower). The preoperative right STA showed a low-resistance flow pattern, different from that in the pediatric case. After surgery, the low-resistance flow pattern remained in the STA. A greater increase in the EDV (290\% increase) than in the PSV (81\% increase) with a decrease in RI (35\% decrease) were also observed at 3 months postoperatively.

\section{Basic Characteristics}

Indirect revascularization surgeries were performed in 50 hemispheres ( 30 patients) in the pediatric group and in 26 hemispheres (19 patients) in the adult group. The basic demographic data are listed in Table 1 . There was no difference in Suzuki stage between the 2 groups. All hemispheres received EDAS surgeries (1 also received EMS in the same craniotomy window), and some hemispheres received additional EPS surgeries in the frontal or parietal region. EDS was performed in every indirect revascularization procedure. The most common surgical procedure in the pediatric group was EDAS only $(60 \%)$, whereas it was EDAS + EPS in the adult group $(69 \%)(\mathrm{p}=0.032)$.

\section{Comparison of Serial Ultrasonographic Values Between the Pediatric and Adult Groups}

The consecutive hemodynamic values of the ultrasonographic parameters in the 2 age groups are shown in Fig. 3. Before surgery, adult patients had a higher mean

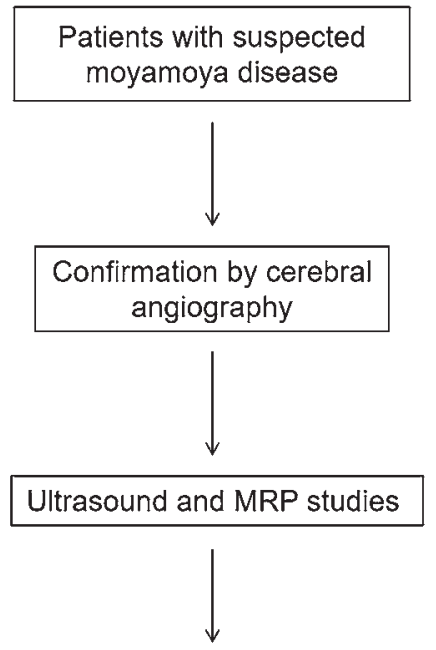

Indirect revascularization* to hypoperfusion area: EDAS only (temporal region), EDAS + EMS (temporal region), or EDAS + EPS (more than temporal region)

- Ultrasound study $(1,3$, and 6 months after surgery $)$

- MRP study (1, 3, 6, and 12 months after surgery)

- Cerebral angiography (before surgery on the contralateral side and 1-2 years after the surgery on the contralateral side)

FIG. 1. Study protocol. After confirmation of MMD by cerebral angiography, patients underwent ultrasonographic examinations and MR perfusion (MRP) imaging. Indirect revascularization procedures were designed according to the hypoperfusion area on MR perfusion images. Ultrasonographic examinations, MR perfusion imaging, and angiographic studies were performed postoperatively. *All of the indirect revascularization procedures were combined with EDS.

EDV (STA: $22.4 \pm 9.5 \mathrm{~cm} / \mathrm{sec}$ vs $12.8 \pm 9.6 \mathrm{~cm} / \mathrm{sec}, \mathrm{p}<$ 0.0001 ; ECA: $20.1 \pm 11.7 \mathrm{~cm} / \mathrm{sec}$ vs $12.2 \pm 6.7 \mathrm{~cm} / \mathrm{sec}$, p $=0.0002)$ and lower mean RI (STA: $0.75 \pm 0.08$ vs 0.85 $\pm 0.07, \mathrm{p}<0.0001$; ECA: $0.80 \pm 0.06$ vs $0.88 \pm 0.07$, p < $0.0001)$ in the STA and ECA, as well as larger FV in the ECA compared with the pediatric group $(153.3 \pm 70.1 \mathrm{ml} /$ min vs $96.0 \pm 43.7 \mathrm{ml} / \mathrm{min}, \mathrm{p}=0.0001$ ). After surgery, both age groups showed similar trends of increased PSV and EDV, decreased RI, and increased FV in the STA and ECA compared with the preoperative state. In comparison between the 2 age groups at 3 months postoperatively, pediatric patients had a higher PSV $(151.4 \pm 73.5 \mathrm{~cm} / \mathrm{sec}$ vs $114.2 \pm 46.1 \mathrm{~cm} / \mathrm{sec}, \mathrm{p}=0.036)$ and greater FV (49.9 \pm $26.2 \mathrm{ml} / \mathrm{min}$ vs $39.5 \pm 26.2 \mathrm{ml} / \mathrm{min}, \mathrm{p}=0.046$ ) in the STA than adult patients, but the EDV in the STA was similar between groups. The RI of the ECA in adult patients was persistently lower than that in pediatric patients $(\mathrm{p}<0.005$ at all time points). In addition, adult patients had a greater $\mathrm{FV}$ in the ECA preoperatively to 3 months postoperatively than pediatric patients (at 3 months: $202.7 \pm 63.7 \mathrm{ml} / \mathrm{min}$ vs $156.7 \pm 57.8 \mathrm{ml} / \mathrm{min}, \mathrm{p}=0.003)$, but there was no intergroup difference at 6 months. 

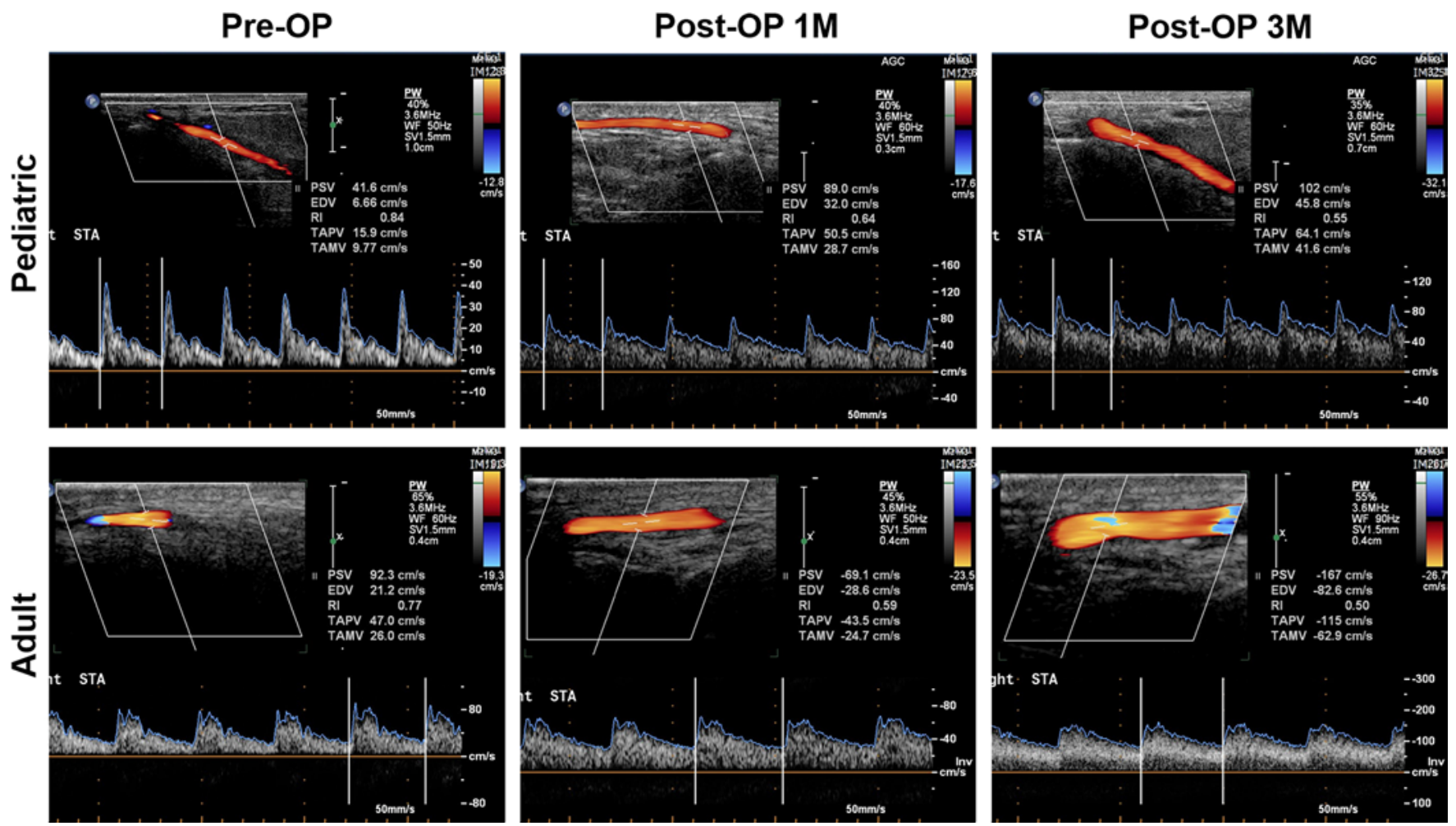

FIG. 2. Ultrasonographic flow patterns of the STAs in 2 representative cases before and after indirect revascularization surgeries. Upper: In a pediatric case, the STA showed a high-resistance flow pattern preoperatively. The STA changed to a low-resistance pattern starting as early as 1 month after surgery. At 1 month postoperatively, an increase in PSV $(41.6 \mathrm{~cm} / \mathrm{sec}$ to $89 \mathrm{~cm} / \mathrm{sec}, 114 \%$ increase) and EDV $(6.66 \mathrm{~cm} / \mathrm{sec}$ to $32 \mathrm{~cm} / \mathrm{sec}, 380 \%$ increase) and a decrease in RI $(0.84$ to $0.64,24 \%$ decrease) were observed. Lower: In an adult case, the STA persistently showed a low-resistance flow pattern before and after surgery. At 3 months postoperatively, both velocity parameters increased (PSV, $81 \%$ increase; EDV, $290 \%$ increase), RI decreased 35\%, and FV increased $247.8 \%$ compared with the baseline. All of the indirect revascularization procedures were combined with EDS. $M=$ month; TAMV = time-averaged mean velocity; TAPV = time-averaged peak velocity. Figure is available in color online only.

\section{Comparison of Consecutive Absolute Changes in the Ultrasonographic Parameters Between Pediatric and Adult Groups}

Because the absolute changes for each ultrasonographic parameter may have a close relationship with the surgeryinduced neovascularization, the absolute changes were compared between the 2 age groups (Fig. 4). Pediatric patients showed larger increases in PSV and EDV in the STA than adult patients $(\triangle \mathrm{PSV}$ at 3 months: $+67.4 \mathrm{~cm} / \mathrm{sec}$ vs $+20.8 \mathrm{~cm} / \mathrm{sec}, \mathrm{p}=0.006 ; \Delta$ EDV at 3 months: $+40.0 \mathrm{~cm} /$ sec vs $+21.1 \mathrm{~cm} / \mathrm{sec}, \mathrm{p}=0.012)$, and also showed a larger PSV increase in the ECA at 3 months $(+23.9 \mathrm{~cm} / \mathrm{sec}$ vs $+9.5 \mathrm{~cm} / \mathrm{sec}, \mathrm{p}=0.025$ ). In addition, the pediatric group showed a more obvious RI decrease $(-0.19$ vs -0.12 , $\mathrm{p}=$ $0.012)$ and a greater FV increase $(+32.8 \mathrm{ml} / \mathrm{min}$ vs +16.9 $\mathrm{ml} / \mathrm{min}, \mathrm{p}=0.001$ ) in the STA at 3 months.

\section{Comparison of Consecutive Percentage Changes in the Ultrasonographic Parameters Between Pediatric and Adult Groups}

Because similar absolute changes in the hemodynamic parameters based on different baseline amounts may result in different hemodynamic impact, percentage changes were compared between the 2 age groups (Fig. 5). Pediatric patients had significantly larger percentage changes in the STA in terms of PSV and EDV at 3 and 6 months and in RI and FV at 3 months postoperatively. For example, pediatric patients had larger PSV percentage changes in the STA at 3 months $(+99.0 \%$ vs $+25.7 \%, \mathrm{p}=0.003)$ and at 6 months $(+139.5 \%$ vs $+42.5 \%, \mathrm{p}=0.009)$ than adult patients. Pediatric patients also showed larger percentage changes in RI $(-21.4 \%$ vs $-15.5 \%, \mathrm{p}=0.047)$ and FV $(+356.7 \%$ vs $+80.7 \%, \mathrm{p}=0.0001)$ in the STA at 3 months.

\section{Comparison of Vessel Diameters Between the Pediatric and Adult Groups}

Vessel diameter is a factor in the equation of flow volume and may differ according to age; thus, the diameters of the STA, ECA, and ICA were compared between the 2 age groups (Supplementary Figure). The diameters of the STA and ICA showed no significant differences in relation to age, but that of the ECA was larger in the adult group preoperatively and at 3 months postoperatively (preoperatively: $3.9 \pm 0.5 \mathrm{~mm}$ vs $3.4 \pm 0.6 \mathrm{~mm}, \mathrm{p}=0.0003 ; 3$ months postoperatively: $3.9 \pm 0.4 \mathrm{~mm}$ vs $3.5 \pm 0.6 \mathrm{~mm}, \mathrm{p}=0.015$ ).

\section{The Determinants of Postoperative Changes of EDV in the} STA

Postoperative changes in EDV ( $\triangle \mathrm{EDV}$ ) in the STA had a positive correlation with collateral grades on angi- 
TABLE 1. Basic demographic data of the moyamoya patients

\begin{tabular}{lccc}
\hline & $\begin{array}{c}\text { Pediatric } \\
\text { Group }\end{array}$ & $\begin{array}{c}\text { Adult } \\
\text { Group }\end{array}$ & $\begin{array}{c}p \\
\text { Value }\end{array}$ \\
\hline No. of hemispheres & 50 & 26 & \\
\hline No. of patients & 30 & 19 & \\
\hline Female sex, $\mathrm{n}(\%)$ & $12(40)$ & $16(84)$ & 0.003 \\
\hline Surgical procedure* & & & 0.032 \\
\hline EDAS only (temporal region) & $30(60)$ & $8(31)$ & \\
\hline EDAS + EMS (temporal region) & $1(2)$ & $0(0)$ & \\
\hline $\begin{array}{l}\text { EDAS + EPS (more than temporal } \\
\text { region) }\end{array}$ & $19(38)$ & $18(69)$ & \\
\hline Suzuki stage* & & & \\
\hline 1 & $1(2)$ & $2(8)$ & \\
\hline 2 & $13(26)$ & $5(19)$ & \\
\hline 3 & $18(36)$ & $5(19)$ & \\
\hline 4 & $10(20)$ & $8(31)$ & \\
\hline 5 & $5(10)$ & $5(19)$ & \\
\hline 6 & $3(6)$ & $1(4)$ & \\
\hline
\end{tabular}

* Data are shown as the number of hemispheres (percentage), and their $p$ values were analyzed by Fisher's exact test.

† Some patients underwent revascularization procedures bilaterally while others had the procedure only on one side. All patients except for one who received bilateral surgery had the same surgical procedure bilaterally, while the one pediatric patient received EDAS + EMS on one side and EDAS only on the other side. All indirect revascularization procedures were combined with EDS.

ography; ${ }^{18}$ thus, a logistic regression model was analyzed to evaluate the determinants of the $\triangle \mathrm{EDV}$ in the STA to search for the factors affecting the postoperative collaterals, including age, sex, preoperative EDV, Suzuki stage, and procedure (stratified by revascularization region: more than the temporal region [EDAS + EPS] vs within the temporal region [EDAS only and EDAS + EMS]) (Table 2). The significant determinant in multivariate analysis for $\triangle E D V$ at 3 months was age (adjusted coefficient $=$ $-0.8, \mathrm{p}=0.030$ ), and those at 6 months were age (adjusted coefficient $=-1.3, \mathrm{p}=0.006$ ) and procedure (adjusted coefficient $=26.5, \mathrm{p}=0.009$ ).

\section{Larger Revascularization Region Resulted in Higher Postoperative Velocities of the STA in Pediatric Patients}

We further compared the flow velocities in the STA after the different procedures (stratified by revascularization region: more than the temporal region vs within the temporal region) in the pediatric and adult groups (Fig. 6). In pediatric patients, the PSV and EDV of the STA after receiving revascularization in more than the temporal region were significantly higher than those within the temporal region at 1-6 months. For instance, the PSV of the STA at 6 months postoperatively was $238.8 \pm 72.4 \mathrm{~cm} / \mathrm{sec}$ after revascularization in more than the temporal region, which was much higher than that after revascularization in the temporal region $(138.6 \pm 55.7 \mathrm{~cm} / \mathrm{sec})(\mathrm{p}=0.001)$. The absolute changes of flow velocities in the STA were also significantly higher in those receiving revascularization in more than the temporal area compared with those within the temporal area in pediatric patients. However, in adult patients, there were no significant differences in the flow velocities or velocity changes between the 2 sets of surgical procedures.

\section{Discussion \\ Greater Postoperative Ultrasonographic Changes in Pediatric Than in Adult Patients}

Although indirect revascularization surgeries can be performed in both pediatric and adult patients, there is debate on whether pediatric patients have better neovascularization than adults after surgery. ${ }^{3,4,16,17,19}$ For example, one report showed that pediatric patients were more likely to develop good collaterals on angiography than adults after EMS surgery, ${ }^{3}$ but another study revealed only a nonsignificant trend of increased STA diameter in pediatric patients compared with adults after indirect revascularization surgeries. ${ }^{4}$ In our previous report, we found that collaterals supplied by ECA on angiography ${ }^{12}$ correlated well with EDV, RI, and FV of the STA and ECA on ultrasonography. ${ }^{18}$ In the present study, we showed for the first time with the aid of ultrasonography that pediatric patients had a significantly higher PSV; greater FV; greater increases in PSV, EDV, and FV; and a greater decrease of RI in the STA and ECA compared with adult patients after indirect revascularization surgeries. Furthermore, this study revealed that age was an independent determinant for postoperative EDV changes, which indicated that age might affect the increased amount of collaterals after surgery. These findings also indirectly support the observations that children receiving indirect revascularization surgeries had more neovascularization on angiography than adult patients. ${ }^{3,17,19}$

\section{Preoperative Age-Related Ultrasonographic Differences}

Before surgery, adult moyamoya patients had higher EDV and lower RI in the STA and ECA than the pediatric group in this study. EDV and RI of these 2 vessels were significantly correlated with the extent of collaterals supplied by them, ${ }^{18}$ so this observation probably indicated that more collaterals might have developed from the STA and ECA before surgery in adult patients. This may partially explain why the onset of symptoms in adult patients was later than that in the pediatric population. This was also compatible with the higher risk of hemorrhagic stroke in adult patients than in the pediatric population due to bleeding from the weak collateral vessels. ${ }^{7}$

\section{Similar Hemodynamic Changes Between Pediatric and Adult Moyamoya Patients After Surgery}

This study revealed that both age groups had similar trends of all hemodynamic parameters on ultrasonography (PSV, EDV, RI, and FV) postoperatively, probably indicating that the collaterals increased in both pediatric and adult groups. Furthermore, there was no significant difference in the postoperative EDV of the STA between adults and children, which was correlated with the postoperative collateral state. ${ }^{18}$ These observations probably indicate that both age groups had increased collaterals after surgery, and the total number of collaterals supplied by the ECA after surgery might be similar between the 2 age 

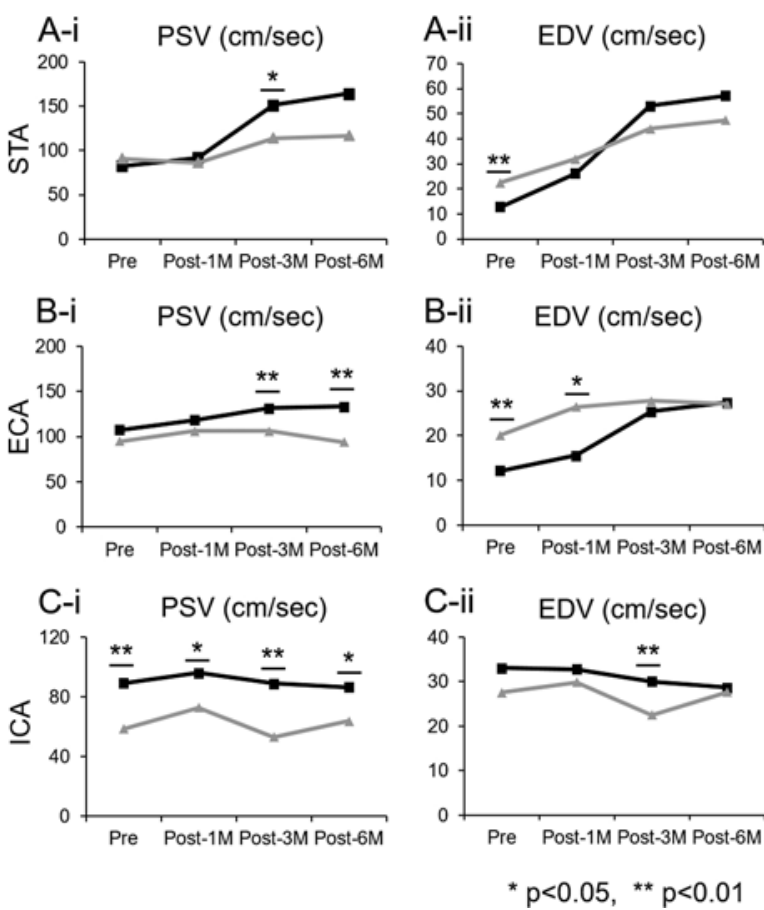
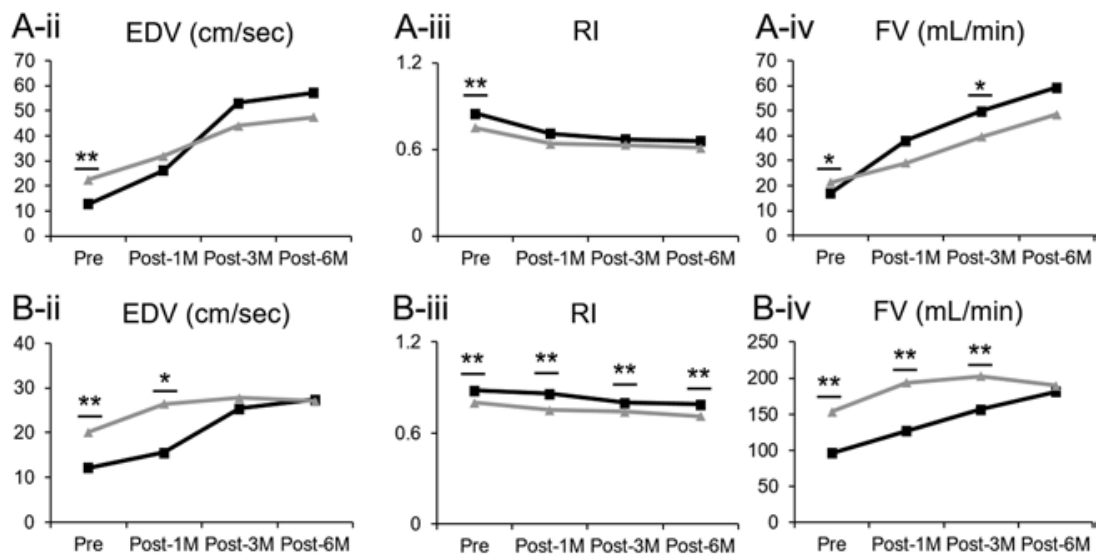

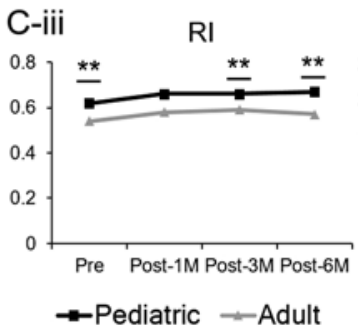

C-iv $\quad \mathrm{FV}(\mathrm{mL} / \mathrm{min})$

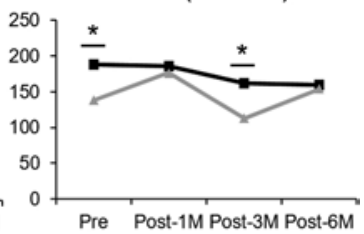

FIG. 3. Comparison of the mean serial ultrasonographic hemodynamic values between the pediatric and adult groups. Before surgery, the adult group had significantly higher EDVs in the STA and ECA ( $p<0.0001$ and $p=0.0002$, respectively) and lower RI (both $p<0.0001$ ) than the pediatric group. After surgery, the pediatric group had a higher PSV (at 3 months, $p=0.036$ ) and a larger FV (at 3 months, $p=0.046$ ) in the STA than the adult group. The EDV and RI in the STA between the 2 groups were similar. In the ICA, pediatric patients had significantly higher PSV, RI, and FV than adults preoperatively and postoperatively (all $p<0.05)$. groups. These findings indirectly support the observation that indirect revascularization surgeries were clinically beneficial in both age groups. ${ }^{1,11,12,17}$

\section{Revascularization Region Affected the EDV of STA in Pediatric Patients}

This study revealed that the size of the revasculariza- tion region was an independent determinant for $\triangle \mathrm{EDV}$ of the STA, which was a potential indicator for postoperative collaterals supplied by the ECA. ${ }^{18}$ This study further showed that, among pediatric patients, a larger revascularization region (more than the temporal region) resulted in higher postoperative EDV and $\triangle \mathrm{EDV}$ in the STA. There were no procedure-related differences in these velocity
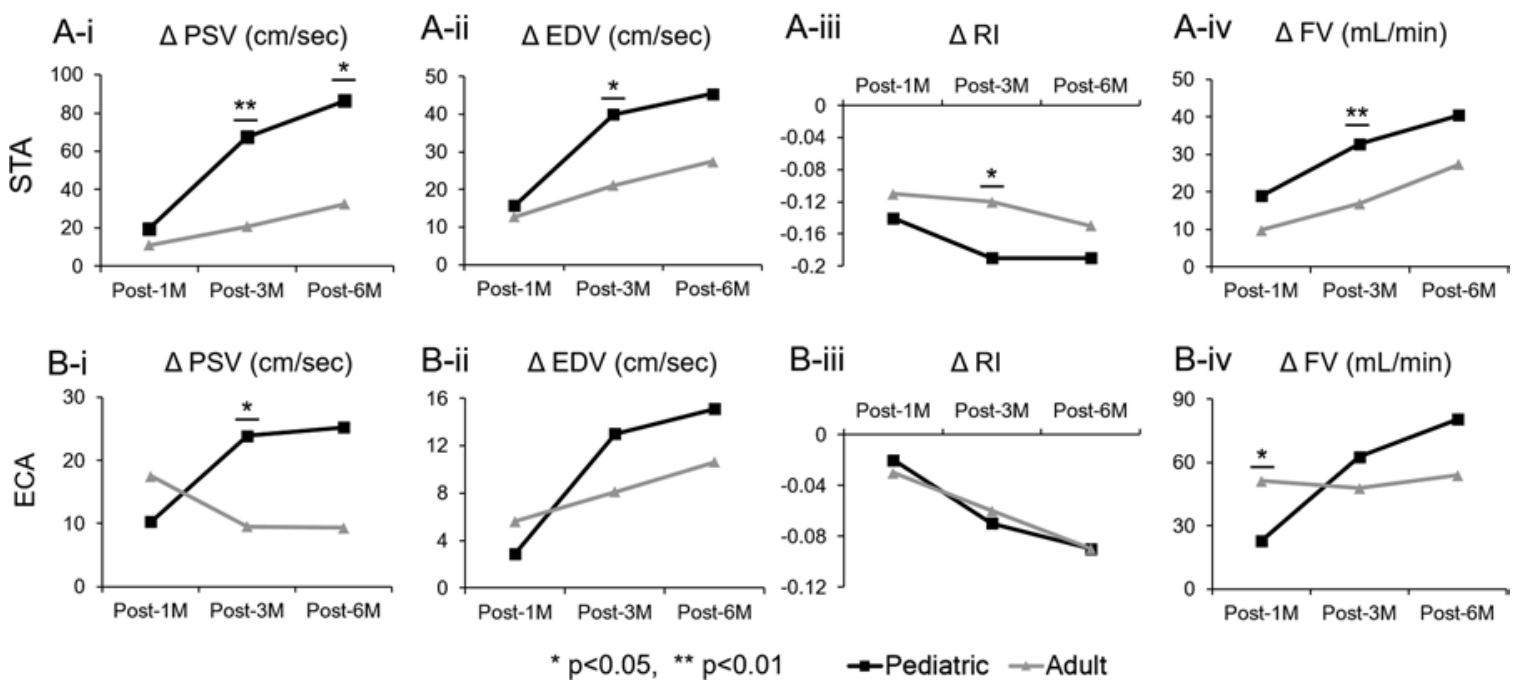

FIG. 4. Comparison of mean absolute changes in serial ultrasonographic parameters between the pediatric and adult groups. Pediatric patients showed greater increases of PSV and EDV in the STA than adult patients at 3 months $(\triangle P S V$ at 3 months, $p=$ 0.006; $\triangle E D V$ at 3 months, $p=0.012)$, a greater PSV increase in the ECA at 3 months $(p=0.025)$, and a greater RI decrease $(p=$ $0.012)$ and a greater FV increase $(p=0.001)$ in the STA at 3 months. 

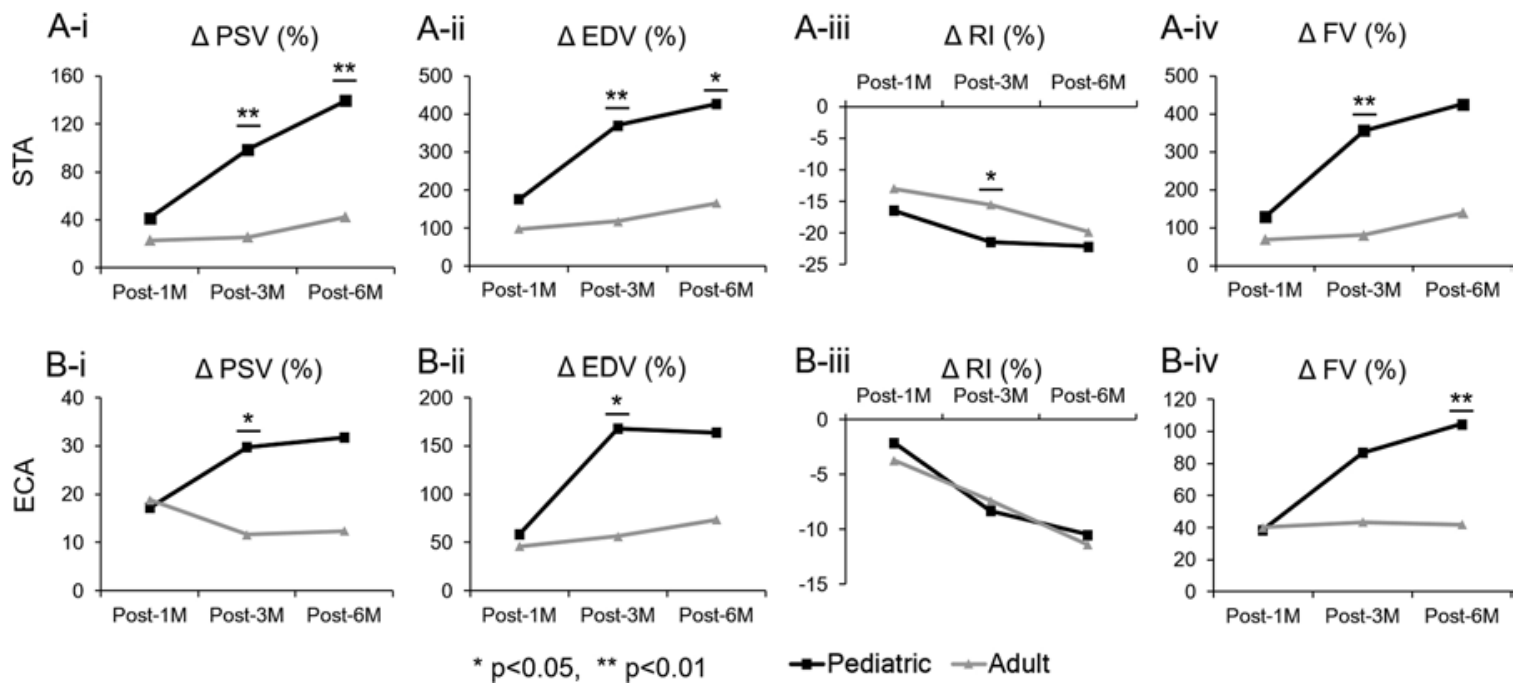

FIG. 5. Comparison of the mean percentage changes of serial ultrasonographic parameters between pediatric and adult groups. Pediatric patients had significantly larger percentage changes in the PSV, EDV, RI, and FV of the STA at 3 and 6 months postoperatively (all $p<0.05$ ). The ECA had a similar trend to that of the STA.

parameters in adult patients, which may be partially due to the small number of hemispheres and relatively short follow-up period. The region of the EPS procedure was designed according to the hypoperfusion region on the MR perfusion scan and on the same side as EDAS. The combination of EDAS surgery with bifrontal EPS had been reported to result in better clinical and angiographic outcomes than EDAS only, especially in the anterior cerebral artery territory. ${ }^{8,10}$ However, these previous reports only focused on pediatric cases, and no prior study has reported the outcomes of EDAS combined with ipsilateral EPS. These findings offered evidence that combined EDAS with ipsilateral EPS may have more benefit than revascularization procedures within the temporal region, especially in pediatric patients.

\section{Study Limitations}

This study suffered from several limitations. First, this study included hemispheres that were treated using more than one kind of indirect revascularization procedure.

TABLE 2. Logistic regression analysis for the determinants of postoperative changes of EDVs in the STA

\begin{tabular}{|c|c|c|c|c|c|c|}
\hline & \multicolumn{3}{|c|}{ Univariate } & \multicolumn{3}{|c|}{ Multivariate } \\
\hline & Coefficient & p Value & $95 \% \mathrm{Cl}$ & Coefficient & $p$ Value & $95 \% \mathrm{Cl}$ \\
\hline \multicolumn{7}{|l|}{$\Delta \mathrm{EDV}$ at $1 \mathrm{mo}$} \\
\hline Age & -0.2 & 0.431 & -0.8 to 0.4 & -0.3 & 0.399 & -1.0 to 0.4 \\
\hline Sex (female vs male) & 1.0 & 0.874 & -11.6 to 13.6 & 4.3 & 0.607 & -12.6 to 21.2 \\
\hline Procedure* & 9.6 & 0.273 & -7.9 to 27.2 & 14.5 & 0.205 & -8.4 to 37.3 \\
\hline Preop EDV & -0.7 & 0.148 & -1.6 to 0.2 & -1.0 & 0.061 & -2.0 to 0 \\
\hline Suzuki stage & 2.3 & 0.410 & -3.3 to 7.8 & 1.5 & 0.630 & -4.7 to 7.6 \\
\hline \multicolumn{7}{|l|}{$\Delta \mathrm{EDV}$ at $3 \mathrm{mos}$} \\
\hline Age & -0.8 & 0.005 & -1.4 to -0.3 & -0.8 & 0.030 & -1.5 to -0.1 \\
\hline Sex (female vs male) & -4.3 & 0.585 & -19.9 to 11.3 & 4.2 & 0.604 & -12.0 to 20.5 \\
\hline Procedure* & 4.4 & 0.579 & -11.2 to 19.9 & 12.9 & 0.106 & -2.8 to 28.6 \\
\hline Preop EDV & -0.8 & 0.035 & -1.5 to -0.1 & -0.7 & 0.091 & -1.5 to 0.1 \\
\hline Suzuki stage & 3.1 & 0.314 & -3.0 to 9.2 & 4.6 & 0.118 & -1.2 to 10.5 \\
\hline \multicolumn{7}{|l|}{$\Delta$ EDV at 6 mos } \\
\hline Age & -0.8 & 0.050 & -1.6 to 0 & -1.3 & 0.006 & -2.3 to -0.4 \\
\hline Sex (female vs male) & 5.6 & 0.558 & -13.5 to 24.7 & 20.4 & 0.052 & -0.2 to 41.0 \\
\hline Procedure* & 22.2 & 0.027 & 2.6 to 41.8 & 26.5 & 0.009 & 7.1 to 45.9 \\
\hline Preop EDV & -0.4 & 0.431 & -1.6 to 0.7 & -0.5 & 0.411 & -1.6 to 0.7 \\
\hline Suzuki stage & 2.1 & 0.599 & -5.9 to 10.2 & -0.1 & 0.975 & -7.5 to 7.3 \\
\hline
\end{tabular}

Boldface type indicates statistical significance.

* Stratified by the revascularization area (more than the temporal region vs within the temporal region). 

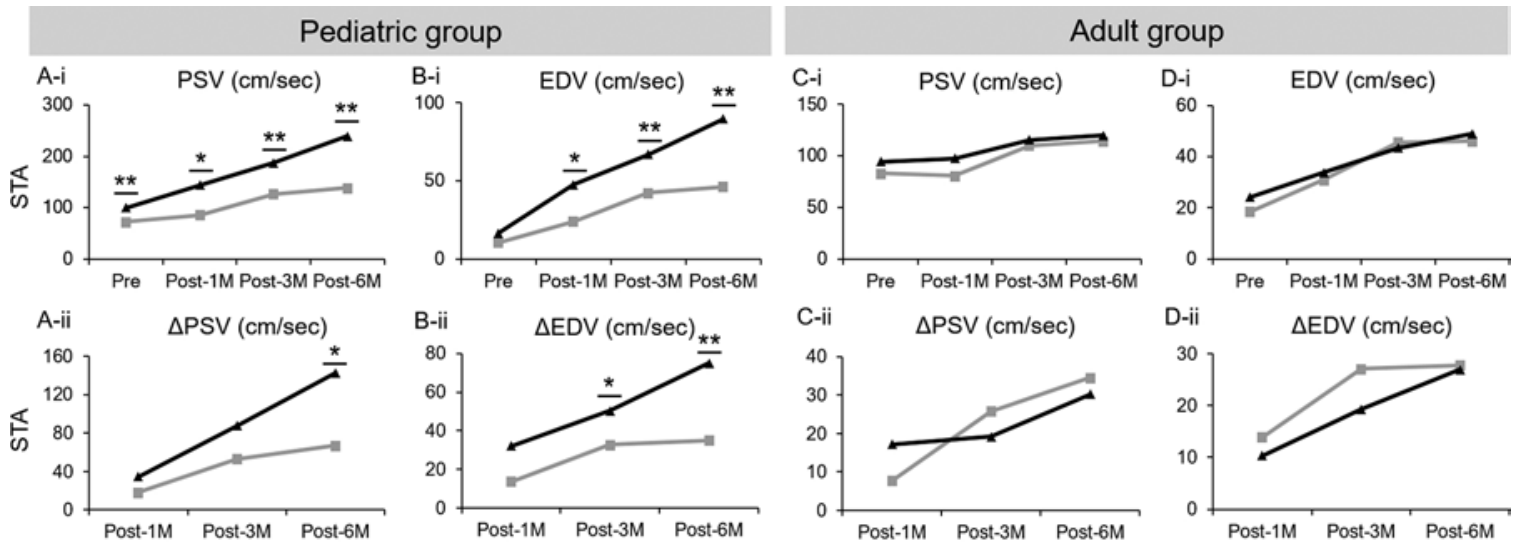

- -Temporal area procedures

-More than temporal area procedures

FIG. 6. Comparison of the mean flow velocities of the STA between different surgical procedures (based on the size of the revascularization region: more than the temporal region vs within the temporal region) in the pediatric and adult groups. In pediatric patients, the flow velocities and the changing velocities in those receiving revascularization in more than the temporal region were significantly higher than those within the temporal region at 1-6 months (all $p<0.05$ ). In adult patients, there was no significant procedure-related difference in the flow velocities.

However, all hemispheres were treated with EDAS, and the neovascularization of the other combined indirect revascularization procedures was developed through similar transdural and transpial routes, which were supplied by the STA and ECA. Second, the number of hemispheres in the adult group was relatively small $(n=26)$, which may reduce the significance of a larger revascularization area in this subgroup analysis. Third, although these ultrasonographic parameters were correlated with the collaterals from the ECA on angiography, ${ }_{18}$ these parameters were indirect indicators for collaterals, and careful interpretation is suggested. The strength of this study is that this is the first report to use ultrasonography to quantify the hemodynamic changes before and after indirect revascularization surgery in relation to age, which may reflect the extent of neovascularization.

\section{Conclusions}

This study showed that pediatric moyamoya patients had significantly greater changes in ultrasonographic parameters in the STA and ECA after indirect revascularization surgery than adult patients, and there were similar postoperative EDVs in the STA between the 2 groups. These observations are consistent with previous angiographic studies that pediatric patients gained more benefit from the indirect revascularization procedures than adults, although the clinical results were satisfactory in both age groups.

\section{Acknowledgments}

We would like to thank Ying-An Chen for performing ultrasound examinations.

Funding was provided by the Ministry of Science and Technology, Taiwan, grant no. 105-2314-B-002-004-MY2 received by Meng-Fai Kuo and grant no. 103-2321-B-002-032 received by Ya-Fang Chen, both for maintaining the study; and by the National Taiwan University Hospital, Taiwan, grant no. 107-M4016 received by Shin-Joe Yeh, for English editing of this manuscript.

\section{References}

1. Agarwalla PK, Stapleton CJ, Phillips MT, Walcott BP, Venteicher AS, Ogilvy CS: Surgical outcomes following encephaloduroarteriosynangiosis in North American adults with moyamoya. J Neurosurg 121:1394-1400, 2014

2. Chen PC, Yang SH, Chien KL, Tsai IJ, Kuo MF: Epidemiology of moyamoya disease in Taiwan: a nationwide populationbased study. Stroke 45:1258-1263, 2014

3. Czabanka M, Vajkoczy P, Schmiedek P, Horn P: Age-dependent revascularization patterns in the treatment of moyamoya disease in a European patient population. Neurosurg Focus 26(4):E9, 2009

4. Dusick JR, Gonzalez NR, Martin NA: Clinical and angiographic outcomes from indirect revascularization surgery for moyamoya disease in adults and children: a review of 63 procedures. Neurosurgery 68:34-43, 2011

5. Fung LW, Thompson D, Ganesan V: Revascularisation surgery for paediatric moyamoya: a review of the literature. Childs Nerv Syst 21:358-364, 2005

6. Guzman R, Lee M, Achrol A, Bell-Stephens T, Kelly M, Do HM, et al: Clinical outcome after 450 revascularization procedures for moyamoya disease. Clinical article. J Neurosurg 111:927-935, 2009

7. Jang DK, Lee KS, Rha HK, Huh PW, Yang JH, Park IS, et al: Clinical and angiographic features and stroke types in adult moyamoya disease. AJNR Am J Neuroradiol 35:1124-1131, 2014

8. Kim CY, Wang KC, Kim SK, Chung YN, Kim HS, Cho BK: Encephaloduroarteriosynangiosis with bifrontal encephalogaleo(periosteal)synangiosis in the pediatric moyamoya disease: the surgical technique and its outcomes. Childs Nerv Syst 19:316-324, 2003

9. Kim JS: Moyamoya disease: epidemiology, clinical features, and diagnosis. J Stroke 18:2-11, 2016

10. Kim SK, Wang KC, Kim IO, Lee DS, Cho BK: Combined encephaloduroarteriosynangiosis and bifrontal encephalogaleo(periosteal)synangiosis in pediatric moyamoya disease. Neurosurgery 50:88-96, 2002

11. Kim T, Oh CW, Bang JS, Kim JE, Cho WS: Moyamoya disease: treatment and outcomes. J Stroke 18:21-30, 2016

12. Matsushima T, Fukui M, Kitamura K, Hasuo K, Kuwabara Y, Kurokawa T: Encephalo-duro-arterio-synangiosis in children 
with moyamoya disease. Acta Neurochir (Wien) 104:96102,1990

13. Mukawa M, Nariai T, Inaji M, Tamada N, Maehara T, Matsushima Y, et al: First autopsy analysis of a neovascularized arterial network induced by indirect bypass surgery for moyamoya disease: case report. J Neurosurg 124:1211-1214, 2016

14. Perren F, Horn P, Vajkoczy P, Schmiedek P, Meairs S: Power Doppler imaging in detection of surgically induced indirect neoangiogenesis in adult moyamoya disease. J Neurosurg 103:869-872, 2005

15. Perren F, Meairs S, Schmiedek P, Hennerici M, Horn P: Power Doppler evaluation of revascularization in childhood moyamoya. Neurology 64:558-560, 2005

16. Piao J, Wu W, Yang Z, Yu J: Research progress of moyamoya disease in children. Int J Med Sci 12:566-575, 2015

17. Takanashi J: Moyamoya disease in children. Brain Dev 33:229-234, 2011

18. Yeh SJ, Tang SC, Tsai LK, Lee CW, Chen YF, Liu HM, et al: Color Doppler ultrasonography as an alternative tool for postoperative evaluation of collaterals after indirect revascularization surgery in moyamoya disease. PLoS One 12:e0188948, 2017

19. Uchino H, Kim JH, Fujima N, Kazumata K, Ito M, Nakayama N, et al: Synergistic interactions between direct and indirect bypasses in combined procedures: the significance of indirect bypasses in moyamoya disease. Neurosurgery 80:201-209, 2017

\section{Disclosures}

The authors report no conflict of interest concerning the materi- als or methods used in this study or the findings specified in this paper.

\section{Author Contributions}

Conception and design: Yeh. Acquisition of data: Yeh. Analysis and interpretation of data: Yeh. Drafting the article: Yeh. Critically revising the article: Kuo, Yeh, Tang, Tsai, Lee, Chen. Reviewed submitted version of manuscript: all authors. Approved the final version of the manuscript on behalf of all authors: Kuo. Statistical analysis: Yeh. Administrative/technical/material support: Kuo. Study supervision: Kuo, Tang, Jeng.

\section{Supplemental Information}

\section{Online-Only Content}

Supplemental material is available with the online version of the article.

Supplementary Figure. https://thejns.org/doi/suppl/10.3171/ 2018.6.PEDS18151.

\section{Previous Presentations}

Portions of this work were presented in poster form at the International Stroke Conference, Los Angeles, California, January 24, 2018 .

\section{Correspondence}

Meng-Fai Kuo: National Taiwan University Hospital, Taipei, Taiwan.mfkenator@gmail.com. 